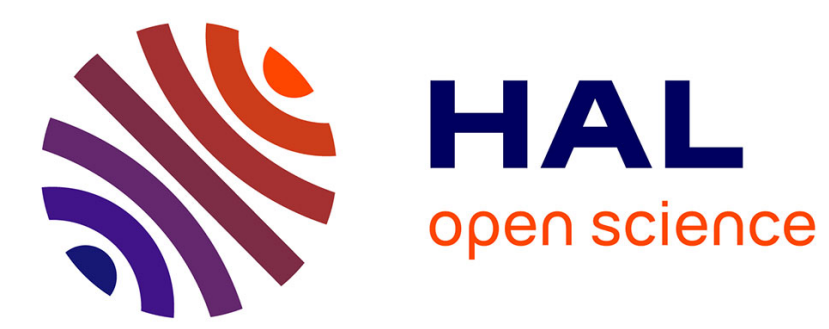

\title{
Réel, contexte, et cognition. Contribution à une histoire de la linguistique cognitive
}

Guy Achard-Bayle, Marie-Anne Paveau

\section{To cite this version:}

Guy Achard-Bayle, Marie-Anne Paveau. Réel, contexte, et cognition. Contribution à une histoire de la linguistique cognitive. Histoire Epistémologie Langage, 2012, XXIV (1), pp.97-114. hal-00773185

\section{HAL Id: hal-00773185 \\ https://hal.science/hal-00773185}

Submitted on 11 Jan 2013

HAL is a multi-disciplinary open access archive for the deposit and dissemination of scientific research documents, whether they are published or not. The documents may come from teaching and research institutions in France or abroad, or from public or private research centers.
L'archive ouverte pluridisciplinaire $\mathbf{H A L}$, est destinée au dépôt et à la diffusion de documents scientifiques de niveau recherche, publiés ou non, émanant des établissements d'enseignement et de recherche français ou étrangers, des laboratoires publics ou privés. 


\author{
Guy ACHARD-BAYLE, Université de Lorraine \& Crem-Praxitexte \\ Marie-Anne PAVEAU, Université de Paris 13 Sorbonne Paris Cité \& Cenel \\ RÉEL, CONTEXTE ET COGNITION
}

CONTRIBUTION À UNE HISTOIRE DE LA LINGUISTIQUE COGNITIVE

\title{
Introduction
}

Suivant une double perspective épistémologique et historique, nous proposons d'interroger dans cette contribution la ou les manières dont la linguistique cognitive de la deuxième moitié du $X X^{e}$ siècle a ou n'a pas pris en compte le contexte dans les opérations mentales de réception (ou d'interprétation) et de production langagières auxquelles elle s'est, dès son fondement, attachée.

Autrement dit, la question que nous voudrions poser est celle de la prise en compte, et du point de vue de son importance ou de son poids et donc du point de vue de son existence, de ce qui relève d'une sinon de la réalité matérielle ; et nous voudrions poser cette question à la fois dans le cadre épistémologique de la cognition linguistique et dans le cadre historique de l'évolution de la linguistique cognitive sur la période définie, de ses débuts chomskyens où réalité et cognition semblent s'ignorer, jusqu'à ses ultimes développements "californiens » où réalité et cognition fusionnent, ou se mélangent(dans les termes du modèle du blending de Fauconnier \& Turner 2002), en réalités conceptuelles.

Pour mener à bien et à son terme un tel parcours, dans une histoire des idées linguistiques et philosophiques aussi foisonnante, nous avons choisi tout d'abord de donner un axe majeur à notre réflexion en nous centrant sur l'opposition internalisme vs externalisme, qui, selon les cadres de référence ou les périodes épistémologiques et historiques, pourra se décliner sous d'autres formes: subjectivisme vs objectivisme, représentationnalisme vs référentialisme; dans le même but, et suivant toujours notre perspective chronologique, nous avons choisi d'«illustrer» ces évolutions et oppositions historiques et épistémologiques, en nous centrant maintenant sur la question dite du mind-body problem.

Notre contribution se présentera en deux grandes parties: dans la première, nous déterminerons la place ou l'existence, et comme nous avons également dit, l'importance ou le poids du contexte dans l'évolution des modèles de linguistique cognitive au travers du dernier demi-siècle; puis à travers la question du mind-body problem, nous réinterrogerons 
la relation du réel à la cognition (et vice versa) en proposant un modèle « réalo-centré », via le contexte, ou plus précisément grâce à lui.

\section{Contexte et cognition à partir de Chomsky}

Pour cette partie historique, nous avons choisi d'aborder et de présenter la question du contexte dans l'évolution des modèles de linguistique cognitivesuivant trois époques. Mais auparavant, nous voudrions apporter quelques précisions disons méthodologiques qui éclaireront nos positions de départ donc nos propositions, puis notre démarche.

Première précision: nous entendons par linguistique cognitive les travaux issus de la « révolution cognitive » américaine des années 1950. Nous admettons bien entendu qu'il existe une cognition antérieure mais nous considérons avec Fuchs 2008 que l'étiquette et le concept de cognition ne sont pas les mêmes au travers des époques, une des différences essentielles entre elles, et une des «marques de reconnaissance » de la période retenue étant la métaphore de l'ordinateur et la notion de computation. Cette linguistique cognitive, comme champ spécifique constitué, a pris ses marques, épistémologiques et théoriques, à partir des conférences de $1956^{1}$, autour de Chomsky, Miller, Simon et Minsky.

Seconde précision : le contexte, tel qu'il apparaît notamment dans notre titre, c'est-à-dire tel qu'il est posé entre les deux autres notions clés, doit de ce fait être justifié ; autrement dit, il nous faut avant tout répondre à la question de savoir pourquoi nous l'avons choisi comme thème et vecteur d'analyse des grands courants de linguistique cognitive dans les soixante dernières années. Nous avancerons quatre raisons :

La première raison est que le contexte est une notion la plupart du temps admise comme « allant de soi » (voir infra notre analyse de Chomsky 2005 [2000]) ; c'est dire qu'on le trouve à peu près dans tous les modèles, y compris, ce n'est pas le moindre des paradoxes, les moins contextualistes (là encore cf. Chomsky).

Une autre raison est que, pour en rester au corpus en français (Schmoll 1996, Achard-Bayle 2006), les auteurs, qui se penchent dans ces recueils sur le « contexte » en tant que tel sont unanimes : le contexte est omniprésent, mais il est, inversement, une notion peu prise en

\footnotetext{
${ }^{1}$ À la fin de l'été 1956, le "MIT Symposium on Information Theory ", qui se tient sur le campus du Darmouth College, rassemble les personnalités les plus marquantes qui ont participé à ce qui est usuellement présenté comme la « révolution cognitive »: la linguistique et la psychologie, ainsi que des domaines qui leur sont liés, se redéfinissent sous l'influence de l'intelligence articielle et des neurosciences émergentes.
} 
compte en tant que telle, et à géométrie variable (cf. Crévenat-Werner ou Kerbrat-Orecchioni in Schmoll 1996, et Chabrolle-Cerretini ou encore Kleiber in Achard-Bayle 2006).

La troisième raison est historique, ce qui cadre bien avec notre perspective : on constate un appel massif au contexte, autrement dit un appel au contexte par des linguistes cognitivistes de plus en plus nombreux depuis les années 80 , sous l'impulsion des paradigmes de la cognition sociale qui sont désormais solidement installés dans les sciences sociales ; il y a eu un «tournant social et culturel » de la cognition générale puis linguistique, avec des évolutions et des modifications notables dont nous voulons rendre compte ici.

Enfin, si nous choisissons le contexte comme fil conducteur de notre analyse à venir dans les sections à suivre, c'est qu'il représente pour nous l'élément clé du modèle cognitif que nous présenterons dans seconde partie.

Nous pouvons maintenant passer aux trois périodes que nous avons retenues pour l'historique de cette première partie.

\subsection{Le Chomskysme}

Dans ses premiers développements américains, c'est-à-dire le cadre chomskyen représentationnel internaliste, le contexte est absent. Le système prédomine et l'activité langagière est associée (i) à un traitement d'informations, (ii) au postulat d'une grammaire universelle.

II n'est toutefois pas inutile de rappeler que Chomsky a toujours voulu situer ou contextualiser sa contribution dans l'histoire des idées (cf. Chomsky 1969 [1966]). Dans l'un de ses derniers ouvrages, Chomsky 2005 [2000] revendique clairement son inscription idéaliste, au sens cartésien du terme, et participe ainsi au débat contemporain du mind-body problem. Ceci dit, la revendication de Chomsky, en rupture avec le référentialisme (voir plus bas son opposition à Putnam), est plus exactement celle de l'internalisme, d'où son fameux I-language: I comme Interne, mais aussi comme Individuel; ce que l'on peut mettre en rapport avec sa défense de l'innéisme et l'intérêt qu'il porte davantage au langage qu'aux langues, ou encore davantage à la grammaire universelle qu'à la diversité des réalisations linguistiques (cf. Fuchs 1997, p. 6).

À partir de ces premières données, fragmentaires mais établies, il est difficile de voir en Chomsky un défenseur du contexte. Ainsi Geeraerts (2003, p. 3 de la version en ligne) fait le lien entre Chomsky et la « décontextualisation de la grammaire ». On peut le vérifier grâce aux outils de recherche numériques, en faisant par exemple le compte exact des occurrences du terme contexte dans le dernier ouvrage de Chomsky : il y en a vingt-cinq, dont la plupart sont des emplois « ordinaires » (voir annexe 1) ; ce constat est conforté par l'examen de l'index (voir annexe 2). 
Toutefois, dans notre relevé des occurrences de contexte, pour l'essentiel ordinaires, la $6^{\text {ème }}$ est une occurrence savante ou spécialisée du terme ; elle est tout à fait intéressante pour notre propos : en effet, dans l'extrait suivant du ch. 2 consacré au « naturalisme » (c'est à dire à l'étude scientifique du langage comme objet d'une science « naturelle »), Chomsky vise un « externalisme non naturaliste » (essentiellement représenté par Putnam ${ }^{2}$ ) :

[...] and non naturalist externalism that attempts to treat human action (referring to or thinking about cats, etc.) in the context of communities, real or imagined things in the world, and so on.

[...] externalisme non-naturaliste qui tente de traiter les actions humaines (référer ou penser aux chats, etc.) dans le contexte de communautés, ou d'objets du monde réels ou imaginaires, etc. (Chomsky 2000, p. 36 ; trad. 2005, p. 106)

Ainsi, cet extrait non seulement illustre le non-contextualisme de Chomsky, mais il est aussi par anticipation une contre-illustration de nos propositions. On peut avoir du mal, dans ces conditions, à concilier de telles positions au sein d'un courant ou d'une histoire du cognitivisme : on peut le vérifier par l'analyse du naturalisme et de l'internalisme de Chomsky que propose Rouveret (2004, pp. 29 sq.) :

La position soutenue par Chomsky depuis la fin des années 1950, réaffirmée dans les articles réunis dans [Chomsky 2000], consiste à défendre la légitimité de la "linguistique interne ». Est dite «interne» une approche qui prend les énoncés de la langue comme autant de phénomènes autonomes dont on peut isoler, étudier et représenter les propriétés structurales et la signification hors des caractéristiques du contexte situationnel, discursif et sociolinguistique dans lequel il prend place. Dans cette conception, le langage n'est pas un objet public et social, dont les locuteurs individuels n'auraient qu'une connaissance partielle, mais un objet strictement individuel, dont la connaissance est interne à l'esprit humain. Ce qui, selon Chomsky, doit intéresser le linguiste, c'est l'étude de cet objet mental, une entité [...] qui doit être prise comme un aspect du monde pouvant être soumis à une enquête rationnelle. Plus précisément, le langage est un objet « psychologique », composante de l'esprit humain, physiquement représenté dans le cerveau, et faisant partie de l'équipement biologique de l'espèce, qui, précisément parce qu'il est ce type d'objet, peut et doit être analysé suivant les mêmes méthodes d'investigation que celles qui sont en œuvre dans les sciences de la nature. » (Rouveret 2004, p. 30 ; nous soulignons)

Pour compliquer encore les choses, et ce sera le dernier mot de cette première section, on voit que le naturalisme internaliste mentaliste de Chomsky est un objet scientifique à facettes difficilement conciliables, si l'on s'en tient aux caractérisations épistémologiques binaires habituelles; en tout cas, le naturalisme en question, pour internaliste et mentaliste, ou

\footnotetext{
${ }^{2}$ Dans un autre genre, non référentialiste, cf. l'extrinsécalité de Cadiot \& Visetti, Nemo et d'autres chercheurs encore, qui se définit, notamment, par son anti-cognitivisme, non mentaliste, et non internaliste... (cf. Achard-Bayle 2007a et b).
} 
encore anti-référentialiste qu'il soit, n'en est pas moins revendiqué comme un réalisme puisqu'il repose sur une étude objective, autrement dit d'un objet proprement matériel, ou matérialisable en termes d'observables.

\subsection{De la Generative Grammar à la Cognitive Linguistics}

À partir des années 1970, toujours aux États-Unis, émerge quelque chose de l'ordre du contextuel et du culturel.

Selon Geeraerts (art. cité 2003), la grammaire générative décontextualisée et désémantisée des années 60 provoque une série de guerres en linguistique (selon l'expression de Harris 1993). Parallèlement, diverses sous-disciplines émergent qui réhabilitent łe sens et/ou le contexte :

Sociolinguistics as we know it now (including the sociology of language, the ethnography of speaking, and sociohistorical linguistics, next to sociolinguistics in the narrow, Labovian sense) [...] pragmatics [...] formal semantics... (Geeraerts art. cité en ligne, p. 5)

Pour autant, toujours selon Geeraerts (art. cité, pp. 5-6), celles-ci côtoient plutôt qu'elles ne tentent d'amender le mainstream chomskyen :

In short, the decontextualising, autonomist attitude of generative grammar is to some extent compensated by the development of disciplines that explore the aspects of language that are relegated to the background by generative grammar. None of the approaches mentioned here, however, overcomes the autonomist restrictions in any fundamental sense. Sociolinguistics and pragmatics exist alongside grammatical theory rather than interacting with it intensively, and the conception of meaning that lies at the basis of formal semantics is too restricted to consider it a truly recontextualised grammar. In other words, the recuperation of the contextual aspects rejected by generative grammar could go further, and this is exactly what is happening in a number of contemporary trends in linguistics.

La linguistique cognitive, telle qu'elle apparaît aux États-Unis à la fin des années 70, sera en effet davantage une réaction, re-contextualisante ou pro-contextualiste, qu'un prolongement aux modèles générativistes, dans lesquels on inclut ici la sémantique générative de Lakoff, et dont Lakoff se débarrasse au profit de la linguistique cognitive de seconde génération : voir Victorri (2004, p. 73 et note 1) ; voir aussi Newmeyer (1986, p. 138) qui souligne, pour sa part, qu'un certain nombre d'idées des travaux ultimes de la sémantique générative ont été incorporés à la seconde linguistique cognitive, mais souvent sans citation !

Remontons maintenant un peu dans le temps, avant l'éclosion de ce que Victorri (2004, p. 73) appelle les «grammaires cognitives» (nous reviendrons ensuite). Cette rapide remontée dans le temps a pour but de nous rappeler : 
1. que c'est avec l'émergence dans les années 70 de la linguistique fonctionnelle et « relationnelle » (Halliday \& Hasan 1976, analysé par Charolles \& Combettes 1999) que sont introduites les notions ou les dimensions discourse-text comme cadres d'analyse, au-delà donc des phrases décontextualisées des modèles grammaticaux structuraux syntaxiques alors dominants ; mais en l'occurrence, c'est davantage de cotexte (et de cohésion) que de contexte (et de cohérence) qu'il s'agit ;

2. que, plus ou moins au même moment, apparaît, en Europe, un courant néogénérativiste qui tente d'appliquer les principes génératifs au texte : il s'agit de la «grammaire de texte » dont Van Dijk, Dressler, Petöfi sont les figures de proue sans parler des «fonctionnalistes de la phrase » de l'École de Prague, tel Daneš 3 .

3. Mais cette grammaire de texte évolue dans les années 80 vers la linguistique textuelle, modèle qui intègre toujours plus (via par ex. la sémantique du discours chez Van Dijk, l'analyse textuelle des discours chez Adam) dimensions pragmatiques, sociales et contextuelles des productions linguistiques, bien au-delà donc du cotexte.

4. Ces travaux, notamment dans leur dimension textualiste, c'est à dire s'orientant vers les genres, se rapprochent de ceux menés en psychologie (aujourd'hui en psychologie cognitive des textes) qui récupèrent des notions diversement contextuelles, certes, mais qui font, toutes, appel ou référence à des communautés cognitives (modèles partagés) : cadres (frames), scripts, scénarios ${ }^{4}$, mais également prototypes $^{5}$.

Revenons maintenant à ce Victorri appelle, au début de son chapitre, les «grammaires cognitives » californiennes, soit « un courant de recherches né dans les années $1980^{6}$, sur la côte Ouest des États-Unis» (ch. cité 2004, p. 73). Malgré le pluriel attaché à «grammaires », Victorri rassemble bien en « un courant " Talmy et Langacker d'un côté, Lakoff et Fauconnier de l'autre, avant néanmoins de les distinguer dans la suite de son étude. On peut en effet considérer les deux premiers comme davantage soucieux de représentation formelle ou syntaxique du sens, quand les deux autres sont davantage, et plus ambitieusement, marqués par le contexte (l'environnement ou les origines) de la conceptualisation : on pense alors particulièrement aux tandems que forment respectivement Lakoff \& Johnson 1999 pour leur théorie de l'embodied mind, philosophie incarnée, et Fauconnier \& Turner 2002 pour leur théorie de l'intégration conceptuelle ou du mélange

\footnotetext{
${ }^{3}$ Voir notamment les contributions d'Adam et Pesek in Achard-Bayle (à par.).

${ }^{4}$ Fillmore 1976, Minsky 1975, Shank \& Abelson 1977, Tannen 1979. Voir la mise en perspective historique de ces concepts in Nehrlich \& Clarke 2000.

${ }^{5}$ Lakoff 1987, Langacker 1987a, Kleiber 1990.

${ }^{6}$ |l vaudrait sans doute mieux dire « qui s'est développé » que « né »; voir infra.
} 
(blending) qui repose sur une anthropologie de l'imagination et une histoire du développement des facultés créatrices chez l'humain ${ }^{7}$.

Ceci dit, il y a effectivement un continuum entre les divers courants apparus dans les années 80 ...Ceci dit, entre les divers courants qui sont apparus dans les années 70 et se sont développés dans les années 80 , il y a effectivement un continuumqui pourrait être assuré par la notion même de conceptualisation, comme on va le voir. Pour autant, cette notion ne nous rapproche pas en tant que telle de la contextualisation qui nous préoccupe comme caractérisant ou non la linguistique cognitive depuis lors. II faut donc essayer de creuser la question plus avant...

Suivant les multiples déclarations programmatiques des linguistes cognitivistes eux-mêmes, on peut, comme le fait Geeraerts (art. cité, pp. 6-7), caractériser la linguistique cognitive par un petit nombre de principes ; et même très exactement deux (qui correspondent aux deux courants distingués plus haut) :

Second, the conceptualizations that are expressed in the language have an experiential basis, i.e. they link up with the way in which human beings experience reality, both culturally and physiologically. In this sense, Cognitive Linguistics embodies a fully contextualized conception of meaning.

Ce second principe nous ramène donc à la problématique qui nous occupe ici. On y trouve l'essentiel du programme défendu et illustré (notamment sur le terrain de la métaphore) par Lakoff \& Johnson 1985 [1980].

On peut se demander néanmoins, pour nous acheminer vers l'issue de cette section sur la linguistique cognitive de quel type de contextualisation il s'agit dans ces modèles (Lakoff \& Johnson comme Fauconnier \& Turner) : tout d'abord de l'enveloppe corporelle de l'esprit (i. e. de l'activité cérébrale, d'où l'embodied mind) ; ensuite, et très souvent dans la première décennie voire au-delà, de l'espace, avec un grand nombre d'études, du courant « grammatical » notamment, sur les verbes de mouvement, les prépositions spatiales (voir Charolles \& Combettes 1999 sur « La grammaire cognitive de Langacker »; et Victorri 2004 sur Vandeloise) $)^{8}$.

\footnotetext{
${ }^{7}$ Selon ces deux auteurs (op. cit. : 6), trois « I caractérisent l'esprit humain : l'identité, l'intégration, l'imagination. L'identité est ce qu'ils appellent une relation vitale, et on comprend bien qu'elle caractérise l'activité cognitive ou consciente de l'esprit humain en tant que réflexivité ; pour l'imagination, nous y reviendrons en conclusion avec la notion d'esprit littéraire; reste donc l'intégration ou le mélange qui est une « opération systématique de manipulation de formes » (ibid.), qui consiste par ex. en un changement métaphorique, en la création d'êtres hybrides, en la superposition des temps et des lieux : "Sur la photo, Jean a les cheveux bruns ", en raccourcis métonymiques: "Platon est sur l"étagère ", etc.

${ }^{8}$ Précédant et préparant la période considérée, dans les années 70 , voir les travaux sur l'espace de Talmy 1975 ou de Miller \& Johnson-Laird 1976.
} 
Chez Lakoff \& Johnson 1985 [1980], cet « espace » est celui à la fois de l'expérience, de la vie quotidienne, et l'expérience elle-même telle qu'elle est conceptualisée ou configurée métaphoriquement ${ }^{9}$; ce qui en fait un espace d'expérience à la fois physique et culturelle (Lakoff \& Johnson 1985, p. 28).

On trouve donc là un espace contextuel à double caractérisation : matérielle et culturelle, conforme au modèle semi-objectiviste, semi-subjectiviste de Lakoff \& Johnson (op. cit. ; voir aussi Lakoff \& Johnson 1999).

Pour clore cette section, nous dirons maintenant un mot d'évaluation sur la contextualisation dont on vient de parler. La question qui se pose en effet, si l'on prend l'exemple emblématique de la métaphore, est la dimension véritablement ou profondément «contextualiste » de cette linguistique cognitive-là. Dans ce cadre, beaucoup de travaux sont parus il est vrai sur la métaphore (ou la métonymie) qui portent sur de nombreuses langues-cultures. Mais, de la même manière que la syntaxe générative avait pu illustrer la pertinence de la théorie et de ses règles par la variété des systèmes, de même la linguistique cognitive de la métaphore repose sur des principes transposables et par là conçus comme universels. L'article de Lakoff 1997, entre « universaux [et] variations », est à ce titre révélateur ${ }^{10}$.

On peut donc demander davantage au contexte en matière de cognition ou de conceptualisation située et partagée. Et c'est bien le mouvement qui semble se dessiner au sein même du courant qui a donné tout dernièrement le jour à l'intercultural ou à la crosscultural pragmatics, suivant d'ailleurs un mouvement amorcé par la linguiste des semantic primes Wierzbicka 1991 : c'est ainsi que Kecskes 2004 présente le cultural crossing comme une suite ou un prolongement du conceptual blending, dans son éditorial du $\mathrm{n}^{\circ} 1$ de la revue Intercultural Pragmatics créée, comme la revue Cognitive Linguistics, chez l'éditeur emblématique du courant : Mouton de Gruyter ${ }^{11}$.

\footnotetext{
${ }^{9}$ Langacker 1987b parle de «mouvement abstrait ». Mais cette expression se trouve déjà chez Gruber 1965.

${ }^{10}$ Pour des détails voir Achard-Bayle 2007a ; voir de même le sous-titre « occidentalo-centré » de Lakoff \& Johnson 1999.

${ }^{11}$ À ce propos, et à l'issue de ce panorama pour la plus grande part américain, il n'est sans doute pas inutile de rappeler les travaux et les propos «pionniers » de Kleiber 2001, p. 367 : « En posant que le mode d'existence ontologique du sens est une propriété émergente [note de l'auteur : voir le numéro 25 d'Intellectica 1997], il est permis [...] de conserver (et donc de pratiquer) une sémantique référentielle ou "réaliste », sans pour autant souscrire à un objectivisme rigide, et d'échapper ainsi, tout en reconnaissant le caractère émergent du sens, à l'instabilité irréaliste d'un constructivisme sémantique échevelé aussi bien qu'à un cognitivisme prédéterminé, étranger à la dimension historique et socioculturelle du sens. »
} 


\subsection{La cognition sera externe ou ne sera pas...}

À partir des années 1980 (et bien avant tant en linguistique qu'en philosophie ou en anthropologie si l'on prend la peine de lire soigneusement et cumulativement ceux que Geerts appelle les "prédécesseurs », comme Bakhtine, Malinowski, Sapir bien sûr, et Vygotski, Peirce, Wittgenstein, etc.), la cognition est pensée comme un système non seulement interhumain mais également environnemental: la cognition emprunte un « tournant social », appuyé sur l'hypothèse de l'externalité de l'esprit, tournant désormais assez bien installé et pris en compte dans les travaux contemporains, comme le montrent ouvrages et colloques consacrés à la question (voir par exemple Brassac 2004, 2006 (dir.), Fiske \& Taylor 2011, Givón 2005, Varela et al. 1993 [1991], Widmer 2010).

La nébuleuse des sciences cognitives étant particulièrement étendue sur les plans disciplinaire et géographique, il est très difficile voire impossible de faire une histoire «événementielle » du tournant social en proposant une date de naissance et un déroulement linéaire. On peut néanmoins proposer quelques balises fortes qui ont fait sortir la cognition de ses encapsulations diverses (esprit, cerveau, neurones, modules, etc.).

Nous proposerons trois moments scientifiques forts qui nous semblent des contributeurs directs à l'externalisation des études sur la cognition linguistique. Ils concernent la cognition générale, et non spécifiquement linguistique, sur laquelle nous revenons infra. II nous semble en effet presque impossible de constituer une histoire de la linguistique cognitive en soi, en tout cas post-chomskyenne, puisqu'elle est étroitement liée aux évolutions des travaux sur la cognition en général qui se répercutent sur l'ensemble des disciplines du champ. Cela explique l'absence des approches que nous présentons ici dans les synthèses sur la linguistique cognitive (par exemple Fuchs (éd.) 2004).

\subsubsection{L'hypothèse de l'extended mind}

L'hypothèse de l'extériorité de l'esprit permet de penser la cognition de manière externaliste, et de « décloisonner » l'esprit, siège des activités cognitives pour le courant dit traditionnel. Encore faut-il préciser de quoi on parle exactement quand on avance cette opposition interne vs externe. Elle peut avoir, comme le précise Lenay 2006, un sens « faible », qui concerne "le contenu intentionnel des représentations ou états mentaux ». Alors que l'internalisme considère que ce contenu est issu des relations internes entre les représentations, l'externalisme y ajoute les « relations causales entre organisme et l'environnement » (Lenay 2006, p. 47). La version forte, dont Hutchins 1995 ou Clark 1997 ou encore Clark \& Chalmers 1998 et Sinha 1988 sont de bons représentants, 
[...] est que les dispositifs techniques ne réalisent pas seulement des modifications artificielles d'une cognition qui serait « naturelle », mais ont un rôle constitutif des activités cognitives pour la perception, le raisonnement, la mémoire, l'imagination ou les interactions.

Ainsi, dans le cadre de la cognition située, l'activité cognitive d'un ou plusieurs sujets peut se comprendre par un jeu d'inscriptions dans le milieu et d'interprétations perceptives [...] de l'organisation spatiale d'objets et de symboles. Dans la dynamique du couplage, les organismes modifient sans cesse la situation partagée en fonction de leur perception de cette situation. Les inscriptions servent de mémoire externe et partageable [...] (Lenay $2006: 47$; nous soulignons).

C'est la version forte que nous adoptons, sans pour autant rejeter les représentations internes, puisque l'extérieur et l'extérieur sont selon nous reliés ou «couplés » et non opposés.

\subsubsection{La cognition sociale}

Nous préférons cette étiquette à celle de constructivisme, qui a le double désavantage de désigner souvent les grammaires cognitives, ou tel ou tel courant comme l'énactivisme ${ }^{12}$ par exemple, et de permettre une opposition binaire qui nous semble réductrice pour les sciences cognitives : constructivisme vs internalisme ou même parfois cognitivisme.

La cognition sociale fédère de nombreux courants très productifs qui se sont développés à partir du début des années 1980 en anthropologie-sociologie (Lave, Suchman, Hutchins, Latour, Quéré, Conein), philosophie (Clark, Dennett, Livet), psychologie (Brassac, Sinha), mais aussi dans le domaine du design (Norman) et de l'écologie (Gibson ${ }^{13}$ ) : les différents choix théoriques dessinent des sous-courants (cognition située, partagée, incarnée, distribuée, etc.), que nous ne détaillons pas. Nous résumons plutôt de manière sommaire et homogène les thèses du champ, dont les implications linguistiques seront détaillées infra (pour une synthèse, voir Clark 1997) :

- les activités cognitives ne sont pas ou pas seulement (il existe un débat entre ceux qui conservent et ceux qui rejettent les représentations) déterminées par des représentations internes individuelles; l'activité cognitive trouve dans l'environnement physique, culturel, historique, etc. différents types de supports matériels ou immatériels, naturels ou artefactuels. L'extérieur est donc considérablement élargi par rapport à l'embodiment neurobiologique des grammaires cognitives ;

\footnotetext{
${ }^{12}$ Courant que nous présentons infra.

${ }^{13}$ Les deux ouvrages de Gibson sur els affordances et sur l'approche écologique de la perception visuelle (voir infra), qui vont irriguer toute la cognition distribuée sont publiés à l'extrême fin des années 1970.
} 
- les données cognitives (informations, savoirs, croyances, etc.) se propagent dans l'ensemble du système cognitif constitué par les individus et les agents non humains de l'environnement ;

- le contrôle des activités cognitives n'est pas centralisé mais " partagé 》 ou « distribué 》 entre les différents agents cognitifs.

\subsubsection{L'énactivisme : penser/parler sans représentations ?}

Troisième approche que nous avons choisie de présenter, la plus connue en France peutêtre grâce aux séjours français et aux publications francophones du biologiste chilien Varela (avec Maturana, également biologiste, voir Maturana \& Varela 1994), l'énactivisme. La théorie de l'énactivisme présente la cognition en train de se faire, c'est à dire comme un «faire-émerger» (ce que formule le néologisme d'énaction), sans appui sur des représentations internes déjà constituées, mais construite au fur et à mesure du déroulement de l'activité cognitive elle-même :

L'idée fondamentale est donc que les facultés cognitives sont inextricablement liées à I'historique de ce qui est vécu, de la même manière qu'un sentier au préalable inexistant apparaît en marchant. L'image de la cognition qui s'ensuit n'est pas la résolution de problèmes au moyen de représentations, mais plutôt le faire-émerger créateur d'un monde avec la seule condition d'être opérationnel : elle doit assurer la pérennité du système en jeu (Varela 1996 [1988]: 111).

La relation entre l'individu et son environnement, en particulier technique, est alors vue comme un « couplage » ou une « codétermination ». Comme le précise Petitmangin : «La perspective énactive, en décloisonnant l'intérieur et l'extérieur, l'esprit et le monde, restaure (ou instaure ?) la possibilité d'une circulation libre entre les deux versants » (2006, p. 91). II s'agit d'une forme d'embodiment plus large que celle proposée par la grammaire cognitive, d'une « inscription corporelle de l'esprit », comme le dit le titre du collectif sur l'énaction de 1991.L'embodiment proposé par la théorie de l'énaction repose en effet sur la définition de la cognition comme l'action de faire émergeren même temps le monde et le sujet, les deux état liés par un "couplage" qui n'est pas simple association ou articulation mais véritable coélaboration. La théorie de l'énaction, dans la version de Varela, possède entre autres, un fondement bouddhiste qui implique une absence de représentation préalable du monde, qui ne vient à l'existence que par le cheminement des agents : "La connaissance ne préexiste pas en un seul lieu ou en une forme singulière ; elle est chaque fois énactée dans des situations particulières. » (Varela et al. 1993 [1991] : 243). Du coup, les connaissance sont incarnées, au sens fort d'inscrites dans la coporéité du sujet : 
Le point central de cette orientation non objectiviste est l'idée que la connaissance est le résultat d'une interprétation permanente qui émerge de nos capacités de compréhension. Ces capacités s'enracinent dans les structures de notre corporéité biologique, mais elles sont vécues et éprouvées à l'intérieur d'un domaine d'action consensuelle et d'histoire culturelle. (Varela et al. 1993 [1991] : 211).

\section{Un tournant contextuel réaliste pour la linguistique cognitive ?}

\subsection{Enjeux linguistiques d'une cognition sociale et culturelle}

Nous voudrions maintenant montrer que l'extrême diversification des sciences cognitives et la perspective de collaboration interdisciplinaire qui se développe actuellement permet aux approches linguistiques de bénéficier des apports d'une cognition sociale et culturelle, en envisageant la cognition humaine comme une activité aux prises avec le réel matériel. Cette perspective implique les trois conséquences suivantes pour la cognition linguistique :

- II existe des « structures cognitives externes » (Auroux 1998) qui demandent de penser la cognition linguistique en relation avec les réalités extérieures. Ces structures cognitives externes peuvent être des outils linguistiques comme les grammaires ou les dictionnaires mais aussi des artefacts et des outils non proprement linguistiques, ni même graphiques, contribuant cependant à la production langagière (du post-it à l'altimètre, en passant par le plan, le bureau ou la machine à café, celle que décrit Lahlou par exemple, dans son article sur les attracteurs cognitifs et le travail de bureau, voir Lahlou 2000). La production langagière n'est donc plus directement interhumaine mais médiée et distribuée.

- Le langagier perd son homogénéité, contre le verbocentrisme de la linguistique cognitive internaliste et des linguistiques non cognitives, même les plus contextuelles comme la linguistique textuelle, l'analyse des conversations ou l'analyse du discours, et devient composite : le langagier est constitué de social, de culturel, d'historique, d'environnemental, de technique, il n'est plus isolé dans sa nature « humaine », l'externalisme signe « la fin de l'exception humaine » (Schaeffer 2007). Nous entendons ici le langagier comme production tant monologale que dialogale ou polylogale : l'idée d'une cognition sociale n'est pas limitée aux interactions dites " sociales ", même si ce sont les situations interactionnelles qui sont surtout prises en compte par les cognitivistes sociaux (Paveau 2009).

- L'opposition systématique réalisme-nominalisme, autrement dit, dans les termes d'aujourd'hui : pro- ou anti- référentialisme / représentationalisme / objectivisme, perd de sa pertinence si l'on considère une voie moyenne : au sens où Larsson 1997, 2008 entend son bon sens commun, et Kleiber 2001, 2006 son intersubjectivité. On voit par ailleurs émerger 
de nouvelles formes de réalismes épistémologiques qui, c'est un signe fort, disséminent en sciences humaines et sociales à partir des études littéraires : ainsi, la géo- et écocritique se développent ${ }^{14}$, dans le fil desquelles on peut mentionner certainesréalités conceptuelles proposées par G. Achard-Bayle 2008 à partir de la Géographie pays invisibles de Giono (Jean le Bleu), définiespar rapport aux faits bruts et (vs) institutionnels de Searle $1995^{15}$.

\subsection{Une linguistique cognitive contextualiste?}

II est intéressant de noter que les propositions linguistiques dans cette perspective ne sont pas faites par des linguistes, qui restent encore très largement, comme nous l'avons dit plus haut, dans la limite du «linguistique homogène », même dans les disciplines les plus contextualistes (pour information on se reportera à Hart \& Lukes 2007 par exemple qui « importent » la théorie californienne de la métaphore en critical discourse analysis sans proposer un programme véritablement cognitif pour l'étude du discours). Ce sont surtout les psychologues qui avancent des hypothèses intéressantes pour une production langagière cognitivement distribuée dans les environnements humains et matériels, dans le cadre interactionniste (ce qui n'est guère étonnant si l'on se rappelle que dans le cadre chomskyen la linguistique est une branche de la psychologie cognitive).

Nous prenons ici l'exemple de Sinha, qui réclame en 2000 « [...] a serious theoreticalmethodological encounter between cultural approaches to human development and cognitive linguistics needs to be put firmly on the agenda » (art. cité, p. 203). II pointe ensuite la nature cognitivo-communicationnelle du langage comme artefact issu de l'organisation socioculturelle de la vie humaine : "Natural language (I suggest) did not emerge, Athenalike, fully armed from the brow of an already-existent human "Mind/Brain". Rather, it emerged as a cognitive-communicative artefact on the basis of human sociocultural organization [...] 》 (art. cité, p. 204). Par conséquent : "In this view, there is no "Mind/Brain", since "mind" is co-constituted by the biological and social » (art. cité, p. 206). Sinha insistera plus tard en 2005 sur le rôle des artefacts matériels dans la production langagière et permettra selon nous une première intégration des objets et des affordances ${ }^{16}$ (propositions d'usage contenues dans les objets eux-mêmes) à un programme linguistique :

\footnotetext{
${ }^{14}$ Ce courant, plus littéraire que linguistique, accorde donc une importance particulière aux environnements, qu'il s'agisse de représentation des lieux dans les textes littéraires ou de l'inscription de la littérature dans l'espace : voir Westphal 2007 et 2011, et le carnet de recherches animé par Michel Collot et Julien Knebusch : http://geographielitteraire.hypotheses.org/tag/geocritique.

${ }^{15}$ Les faits institutionnels sont des artefacts humains (la monnaie ou la valeur d'une monnaie), tandis que les bruts existent ou existeraient sans l'homme même (la hauteur de l'Himalaya).

${ }_{16}$ Le terme affordance est un emprunt direct à l'anglais affordance et a été acclimaté en français sous cette forme. II est proposé dans le sens de « possibilité d'action et d'usage des objets " par J. Gibson dans ses travaux sur la perception (The theory of Affordances en 1977 et The Ecological Approach to
} 
[...] we argue [...] that the key notion of "embodiment" need[s] to be extended beyond its focus on the humanly corporeal, the "brain-in-the-body", to take account of the way that cognitive and cultural schemas find material realization-are embodied-in the artefacts of material culture; and the way in which such artefacts are themselves embedded in culturally appropriate, normative structures of action and interaction. In this perspective, mind is socially distributed between people, and mental processes are supported by objects which embody and represent them. Cognition extends beyond the individual; embodiment goes beyond the skin (Sinha 2005, p. 8 ; nous soulignons).

Dans le champ francophone nous constatons le même type d'inscription disciplinaire : nous citerons les propositions de Brassac par exemple, qui dénonce ce qu'il appelle l'« égocéphalocentrisme» de la linguistique cognitive internaliste mais également du conversationnalisme, et qui présente l'interaction verbale comme une « production cognitive conjointe »: "Production cognitive conjointe, la construction de significations qui trame l'interaction entre sujets humains est une dynamique de modelage de formes langagières, corporelles et artefactuelles» (2004, pp. 251-252). Pour lui en effet, «la création de matérialité est au cœur du dispositif intercognitif que constitue le déploiement de l'interaction (2001, p. 12). Ce qui l'amène à proposer d'articuler sur un continuum l'intersubjectivité et l'interobjectivité (concept proposé par Latour). Ces propositions sont reprises par certains pragmaticiens comme Mondada (par exemple Mondada 2005), toujours dans le cadre interactionniste, et pour l'étude du discours dans son acception anglo-saxonne de conversation et interaction.

Nous ne sommes cependant pas entièrement convaincus par ces propositions dans la mesure où elles ne concernent que l'interaction conversationnelle di- ou polylogale. Comme Geeraerts 2003, nous pensons qu'une linguistique cognitive véritablement contextualisante doit affecter le système linguistique, donc concerner la langue, et par conséquent toutes les productions langagières quels que soient les dispositifs de production, monologaux ou polylogaux, oraux ou écrits, textuels ou discursifs, etc. Nous pensons donc qu'un programme pour une linguistique cognitive contextualiste, que nous appelons « linguistique symétrique » (i.e. qui ne considère pas la relation entre l'esprit et le monde comme asymétrique, c'est la position de B. Latour en anthropologie) doit intégrer quelque chose comme une description linguistique (une «grammaire »), ce qui implique de conserver les représentations internes vus comme lieux d'inscription des fonctionnements langagiers actualisés en contexte. C'est dans cette perspective que nous travaillons, Achard-Bayle proposant le concept de réalité conceptuelle (Achard-Bayle 2008), dont nous venons de parler, et Paveau celui de 
prédiscours (Paveau 2006, 2007, 2011) ${ }^{17}$ et cela implique un certain nombre de révisions, voire de renoncements à la fois théoriques, méthodologiques et psychologiques de la part des linguistes, nous semble-t-il.

\section{Conclusion}

Pour conclure, nous présentons les révisions impliquées selon nous par le programme d'une linguistique cognitive contextualiste, donc symétrique.

Tout d'abord l'opposition binaire entre interne et externe doit être repensée (elle n'est d'ailleurs plus admise comme évidente dans nos disciplines depuis les années 1990) pour permettre d'adopter une position «continuiste» autorisant la circulation tant des représentations, ou des conceptualisations si l'on préfère, que des matérialités entre les deux. Ensuite il nous semble nécessaire de renoncer à l'homogénéité langagière de la langue et des productions langagières et d'admettre son hétérogénéité, c'est-à-dire l'idée que le langagier est de nature composite, ou hybride, car il contient à la fois du langagier et les matérialités environnementales qui le permettent. II nous semble enfin intéressant de réhabiliter une forme de réalisme qui ne se contente pas de dire que la réalité est une construction (sociale ou autre, voir sur ce point Hacking $1999^{18}$, mais d'en tirer un réel parti ; la réalité doit ainsi se situer non seulement à mi-chemin des faits bruts et institutionnels de Searle $1995^{19}$, mais aussi, dans l'esprit (c'est le cas de le dire) du Literary Mind (Turner $1996)^{20}$, comme un composé nécessaire des deux. ${ }^{21}$

\footnotetext{
${ }^{17}$ Les réalités conceptuelles sont des représentations sinon des reproductions mentales d'entités matérielles; elles mêlent donc le matériel à l'immatériel. Elles intéressent les sciences du langage en ce qu'elles sont accessibles par ce que les linguistes cognitivistes (Sweetser \& Fauconnier 1996) appellent des linguistic reflections (voir par ex. Achard-Bayle op. cit., ch. 8 sur « une » ou « des Provence(s) ?»). Les prédiscours sont selon Paveau des cadres prédiscursifs collectifs (savoirs, croyances, pratiques) qui ont un rôle instructionnel pour la production et l'interprétation du sens en discours. Ils sont des opérateurs dans la négociation de la propagation et de la distribution du sens dans les collectivités humaines, des plus restreintes et informelles aux plus larges et instituées. Dans la perspective de la cognition sociale, on les considérera comme des données propagées par une mémoire « cognitivo-discursive » (retravail du concept de "mémoire discursive » de Courtine et de celui de "mémoire interdiscursive » de Moirand), et distribuées par un certain nombre d'agents internes et externes comme les artefacts ou les technologies cognitives.

${ }^{18}$ L'ouvrage est aussi polémique en ce qu'il s'oppose à une tendance antiréaliste des sciences, dans la décennie où il paraît, à voir des constructions sociales partout, donc pas seulement dans les SHS : «Pourquoi ne pas associer la géologie et l'idée de construction sociale ? » (op. cit. : 12). Kleiber, dans divers textes, dont ceux cités, dresse le même constat, et mène le même combat, dans les sciences du langage, où il défend un « réalisme modéré ». Ceci dit, l'interrogation, ironique, de Hacking sur la géologie trouve un écho, inversement ironique, dans les travaux géo- et écocritiques qui se mènent et se développent aujourd'hui en littérature ! Voir supra note 14.

${ }^{19}$ Voir supra note 15.

${ }^{20}$ L'esprit est littéraire pour Turner comme les métaphores sont quotidiennes chez Lakoff \& Johnson (1985 [1980]) : autrement dit les figures ou mieux les figurations fictives ou fictionnelles habitent notre
} 
Références bibliographiques (les références électroniques ont été vérifiées le 30 décembre 2011)

Achard-Bayle, Guy (éd.). (2006) Présentation de "Textes-Contextes", Pratiques 129-130.

Achard-Bayle, Guy (2007a). "Sens, référence... et cognition », in Jean-Rémi Lapaire (éd), Actes du Colloque From Gram to Mind: Grammar as Cognition, Bordeaux, 18-20 mai 2005, Bordeaux, Presses Universitaires, 103-133.

Achard-Bayle, Guy (2007b). "Les réalités conceptuelles et leurs ancrages matériels. Les sémantiques cognitives et la question de l'objectivisme ", Corela, Numéro spécial Cognition, discours, contextes : http://corela.edel.univ-poitiers.fr/index.php?id=1532

Achard-Bayle, Guy (2008). Les réalités conceptuelles. Identité et/en fiction, Publications de l'université Paul Verlaine Metz, coll. Recherches textuelles 8.

Achard-Bayle, Guy (éd.) (à par.). « Linguistique textuelle, états de lieux », Verbum, Nancy, PU.

Adam, Jean-Michel (2005). La Linguistique textuelle. Introduction à l'analyse textuelle des discours, Paris, Arman Colin. (Nouvelles éditions en 2008 et 2011).

Adam, Jean-Michel (à par). "L'émergence de la linguistique textuelle en France (1975-2010). Parcours bibliographique en 100 titres », in G. Achard-Bayle (éd.) (à par.).

Auroux, Sylvain (1998). La raison, le langage et les normes, Paris, PUF.

Brassac, Christian (2001). "Co-responsabilité cognitive et dissolution de frontières ", colloque Des sciences et des frontières, Nancy, 10-12 mai, texte en ligne sur http://www.univnancy2.fr/pers/brassac/publis.html

Brassac, Christian (2004). "Action située et distribuée et analyse du discours: quelques interrogations ", Cahiers de linguistique française 26, 251-268.

Brassac,Christian (dir.) (2006). «Internalisme / Externalisme », Intellectica 43.

Chabrolle-Cerretini, Anne-Marie (2007). "La linguistique cognitive et Humboldt », Corela, Numéro spécial Cognition, discours, contextes : http://corela.edel.univ-poitiers.fr/index.php?id=1476

Charolles, Michel et Combettes, Bernard (1999). «Contribution pour une histoire récente de l'analyse du discours ", Langue Française 121, 76-116.

Chomsky, Noam(1969 [1966]). Lalinguistique cartésienne, Paris, Éditions du Seuil.

Chomsky, Noam(2005 [2000]). Nouveaux horizons dans l'étude du langage et de l'esprit, Paris, Stock.

langage ordinaire, de la métaphorisation et de la dramatisation des énoncés (comme « Le toit a volé en éclats. ") au recours à l'utilisation des proverbes, des paraboles... Voir aussi les opérations de mélange (blending) qui créent souvent du fictif, supra note 7.

${ }^{21}$ Nous remercions Jean-Michel Fortis de sa relecture attentive. 
Clark, Andy (1997). Being There: Putting Brain, Body, and World Together Again, Cambridge (MA), MIT Press.

Clark, Andy and Chalmers, David (1998). «The Extended Mind », Analysis 58, 7-19.

Crévenat-Werner, Danielle (1996). «La notion de contexte dans les dictionnaires », Scolia, 13-38.

Daneš, František (1974). Papers on Functional Sentence Perspective, Academia Publishing house of the Czechoslovak Academy of Sciences, La Hague et Paris, Mouton.

van Dijk, Teun (1972). Some Aspects of Text-Grammars, La Hague, Mouton.

van Dijk, Teun (1977). Text and Context, Londres, Longman.

van Dijk, Teun (1985). "Semantic Discourse Analysis », in T. A. van Dijk et al. (éds), Handbook of Discourse Analysis, vol. 2, Londres, Academic Press, 103-136.

van Dijk, Teun (2006). « Discourse, Context and Cognition », Discourse Studies 8-1, 159-177.

van Dijk, Teun \& Kintsch, Walter (1983). Strategies of Discourse Comprehension, New York, Academic Press.

Dressler, Wolfgang U. (éd.) (1978). Textlinguistik, Darmstadt, Wissenschaftliche Buchgesellschaft.

Fauconnier, Gilles, and Turner, Mark (2002). The Way We Think: Conceptual Blending and the Mind's Hidden Complexities, New York, Basic Books.

Fillmore, Charles (1976). "Frame Semantics and the Nature of Language ", in S.R. Harnad, D.S. Horst \& J. Lancaster (eds), Origins and Evolution of Language and Speech, Annals of the New York Academy of Sciences, Vol. 280, 20-32.

Fiske, Susan, et Taylor, Shelley (2011). Cognition sociale. Des neurones à la culture, Wavre, Mardaga

Fuchs, Catherine (1997). «Diversité des représentations linguistiques: quels enjeux pour la linguistique cognitive ? », in C. Fuchs \& S. Robert (éds), Diversité des langues et représentations cognitives, Gap-Paris, Ophrys, 5-24.

Fuchs, Catherine (2004) (ed.). La linguistique cognitive, Gap-Paris, Ophrys.

Fuchs, Catherine (2004). "Pour introduire à la linguistique cognitive ", in C. Fuchs (ed), La linguistique cognitive, Gap-Paris, Ophrys, 1-24.

Fuchs, Catherine (2008). « Linguistique française et cognition », conférence plénière au CMLF'08, pdf en ligne :

http://www.linguistiquefrancaise.org/index.php?option=com article\&access=doi\&doi=10.1051/cmlf083 $\underline{40 \& \text { Itemid }=129}$

Geeraerts, Dirk (2003). "Decontextualizing and Recontextualizing Tendencies" in 20th-century Linguistics and Literary Theory, in Menge E., Schmid H.-J. \& Steppat M. (eds.), Anglistentag 2002 Bayreuth, Trier, Wissenschaftlicher Verlag, 369-379. 
Giono, Jean (1974 [1932]). Jean le Bleu, Paris, Librairie Générale Française-Le Livre de Poche.

Givón, Thomas (2005). Context as Other Minds. The Pragmatics of Sociability, Cognition and Communication, Amsterdam/Philadelphie, John Benjamins Publishing Company.

Hacking, Ian (2001 [1999]). Entre science et réalité : la construction sociale de quoi ?, Paris, Les Éditions La Découverte.

Halliday, Michael and Hasan, Ruqaiya (1976). Cohesion in English, Londres, Longman.

Harris, Randy (1993). The linguistics wars, Oxford, Oxford University Press.

Hart, Christopher, and Lukes, Dominik (2007) (eds.). Cognitive Linguistics in Critical Discourse Analysis. Application and Theory, Newcastle, Cambridge Scholars Publishing.

Hutchins, Edwin (1995). Cognition in the Wild, Cambridge (MA), MIT Press.

Kecskes, Istvan (2004). "Editorial: Lexical Merging, Conceptual Blending, and Cultural Crossing », Intercultural Pragmatics 1-1, 1-26.

Kerbrat-Orecchioni, Catherine (1996). « Texte et contexte », Scolia, 39-60.

Kleiber, Georges (1990). La Sémantique du prototype, Paris, PUF.

Kleiber, Georges (2006). "Introduction au(x) contexte(s) », Entretien avec G. Achard-Bayle (éd.), Textes-Contextes, Pratiques 129-130,17-19.

Lahlou, Saadi (2000). «Attracteurs cognitifs et travail de bureau », Intellectica 30, 75-113.

Lakoff, George (1987). Women, Fire and Dangerous Things, Chicago, The University of Chicago Press.

Lakoff, George (1997). "Les universaux de la pensée métphorique : variations dans l'expression linguistique », in C. Fuchs \& S. Robert (éds), 165-181.

Lakoff, George, and Johnson, Mark (1985 [1980]). Les Métaphores dans la vie quotidienne, Paris, Éditions de Minuit.

Lakoff, George and Johnson, Mark (1999). Philosophy inthe Flesh. The Embodied Mind and its Challenge to Western Thought, New York, Basic Books.

Langacker, Ronald (1987a). Foundations of Cognitive Grammar. Vol. 1 : Prerequisites, Stanford, Stanford University Press.

Langacker, Ronald (1987b). « Mouvement abstrait », Langue Française 76, 59-76.

Larsson, Bjorn (1997). Le "bon" sens commun. Remarques sur le rôle de la (re)cognition intersubjective dans l'épistémologie et l'ontologie du sens, Études romanes de Lund 57, Lund, Lund university Press.

Larsson, Bjorn (2008). «Le sens commun ou la sémantique comme science de l'intersubjectivité humaine »,Langages 170, 28-40. 
Lenay, Charles (2006). «Énaction. Externalisme et suppléance perceptive », Intellectica 43, 27-52.

Maturana, Humberto, et Varela Francisco (1994). L'arbre de la connaissance, Paris, Eddison-Wesley.

Minsky, Marvin (1975). A framework for representing knowledge, in P.H. Winston (ed.), The Psychology of Computer Vision, New York, McGraw-Hill, 211-277.

Mondada, Lorenza (2005). «Espace, langage, interaction et cognition : une introduction », Intellectica, 41-42, 7-23.

Nerlich, Brigitte \& Clarke, David D. (2000). "Semantic fields and frames: Historical explorations of the interface between language, action, and cognition », Journal of Pragmatics, 32, 125-150.

Paveau, Marie-Anne (2006). Les prédiscours. Sens, mémoire, cognition, Paris, Presses Sorbonne nouvelle.

Paveau, Marie-Anne (2007). "Discours et cognition. Les prédiscours entre cadres internes et environnement extérieur », Corela : "Contextes, discours, cognitions », http://corela.edel.univpoitiers.fr/index.php?id=1550

Paveau, Marie-Anne (2009). "Mais où est donc le sens ? Pour une linguistique symétrique », Actes du colloque international Res per nomen, Reims, 30-31 mai 21-31.

Paveau, Marie-Anne (2011). "Quelles données entre l'esprit et le discours ? Du préconstruit au prédiscours ", dans Azouzi A. (dir.), L'analyse du discours. Notions et problèmes, Tunis, Éditions Sahar, 19-37.

Pešek, Ondřej (à par.). "La linguistique textuelle tchèque au seuil du $X X I^{e}$ siècle: la genèse d'une discipline et la tradition pragoise », in G. Achard-Bayle (éd.) (à par.).

Petitmangin, Claire (2006). «L'énaction comme expérience vécue», Intellectica 43, 85-92.

Petöfi, Sandor (1975). Vers une théorie partielle du texte, Hambourg, Helmut Buske Verlag.

Rouveret, Alain (2004). « Grammaire formelle et cognition linguistique », in C. Fuchs (éd.), 27-71.

Schaeffer, Jean-Marie (2007). La fin de l'exception humaine, Paris, Gallimard.

Schmoll, Patrick (éd.), (1996). Contexte(s), Scolia.

Searle, John (1998 [1995]). La Construction de la réalité sociale, Paris, Gallimard.

Schaeffer, Jean-Marie (2007). La fin de l'exception humaine, Paris, Gallimard.

Shank, Roger, Abelson, Robert (1977). Scripts, Plans, Goals, and Understanding, Hillsdale, Lawrence Erlbaum.

Sinha, Chris (1988). Language and Representation: A Socio-naturalistic Approach to Human Development, Hemel Hempstead, Harvester-Wheatsheaf.

Sinha, Chris (2000). "Culture, Language and the Emergence of Subjectivity », Culture \& Psychology, Vol. 6 (2), London, Thousand Oaks, CA and New Delhi, Sage Publications, 197-207.

Sinha, Chris (2005). "Blending out of the Background: Play, Props and Staging in the Material world », Journal of Pragmatics 37, 1537-1554. 
Sweetser Eve \& Fauconnier Gilles (1996). "Cognitive Links and Domains: Basic Aspects of Mental Space Theory », in G. Fauconnier \& E.Sweetser, Spaces, Worlds and Grammar, Chicago, The University of Chicago Press, 1-28.

Tannen, Deborah (1979). "What's in a frame? Surface evidence for underlying expectations ", in R. Freedle (ed.) New Directions in Discourse Processing, Norwood, Ablex, 137-181.

Turner, Mark (1996). The Literary Mind, Oxford, Oxford University Press.

Vandeloise, Claude (1986). L'espace en français, Paris, Éditions du Seuil.

Varela, Francisco (1996 [1988]). Invitation aux sciences cognitives, Paris, Éditions du Seuil.

Varela, Francisco, Thompson, Evan, et Rosch, Eleanor (1993 [1991]). L'inscription corporelle de l'esprit. Sciences cognitives et expérience humaine, trad. V. Havelange, Paris, Seuil.

Westphal, Bertrand (2007). La Géocritique. Réel, fiction, espace. Paris, Les Éditions de Minuit.

Widmer, Jean (2010). Discours et cognition sociale. Une approche sociologique, Paris, Éditions des archives contemporaines.

Wierzbicka, Anna (1991). Cross-Cultural Pragmatics: The Semantics of Human Interaction, Berlin, Mouton de Gruyter. 


\section{Occurrences de context in Chomsky 2000}

- 1.on Page 3:"... he hears, express his thoughts, and use his language in a variety of other ways. Generative grammar arose in the context of what is often called "the cognitive revolution" of the 1950s, and was an important factor in its development. ..

- 2.on Page 19:"..., but that in the domain where questions of realism arise in a serious way, in the context of the search for laws of nature, objects are not conceived from the peculiar perspectives provided by concepts of common-sense.

- 3.on Page 23:"... In the context of Putnam's discussion, discoveries about the brain at these levels of inquiry are on a par with a discovery about

- 4.on Page 36:"... and nonnaturalist externalism that attempts to treat human action (referring to or thinking about cats, etc.) in the context of communities, real or imagined things in the world, and so on. Such approaches are to be judged on their ..

- 5.on Page 39:"... " It also seems unclear that metaphysical issues arise in this context. To take some of Kripke's examples, doubtless there is an intuitive difference between the judgment that Nixon would be the ..."

\section{Index de Chomsky 2000}

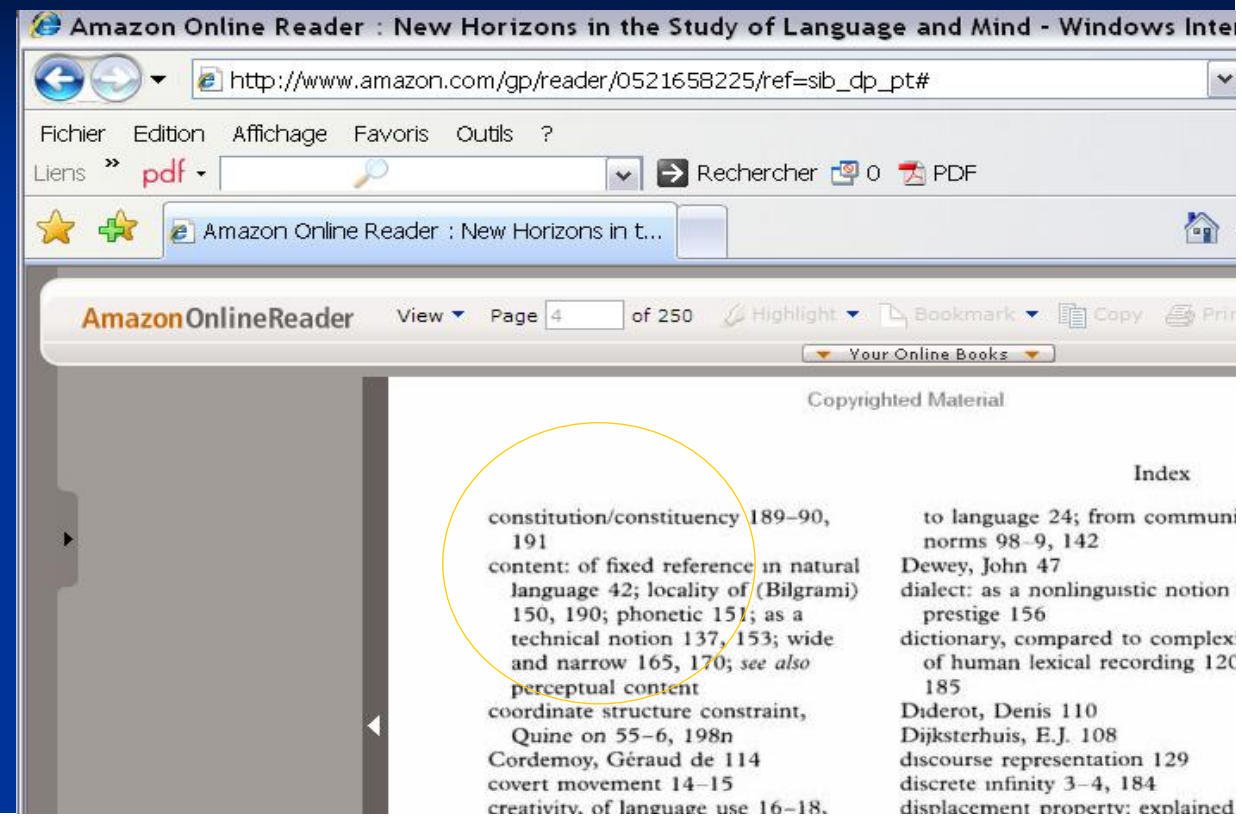




\section{Guy ACHARD-BAYLE, Marie-Anne PAVEAU}

\section{Réel, contexte, et cognition. Contribution à une histoire de la linguistique cognitive}

Cet article interroge, dans une perspective historique et épistémologique, la manière dont la linguistique cognitive du demi-siècle passé a pris en compte (ou non) le contexte dans les opérations mentales de réception / interprétation et de production langagière auxquelles elle s'est, dès son fondement, attachée.

L'axe de réflexion est l'opposition internalisme vs externalisme et la question posée est celle de la prise en compte du poids et de l'existence de ce qui relève, dans la cognition linguistique, de la réalité matérielle. Sont abordés le traitement du contexte dans les linguistiques cognitives depuis Chomsky et celui du mind-bodyproblem dans les courants hétérodoxes de la cognition située à partir des années 1990. Des propositions sont faites pour une meilleure intégration des environnements de la production verbale aux analyses linguistiques dans une perspective cognitive.

Mots clés : contexte, cognition, cognition sociale, externalisme, internalisme, mindbody problem, réalité

\section{The real, context and cognition. A contribution to a history of cognitive linguistics}

The object of this study is to ask how cognitive linguistics has addressed, over the course of the last half century, the role of context in the mental operations of reception/interpretation and language production (operations which are the core foci of its key concerns from the very outset of cognitive linguistics).

The more specific focus of our study is the conflict between internalism and externalism, in other words the significance given to material reality in linguistic cognition. We will discuss the treatment of the notion of context in cognitive linguistics since Chomsky, and the way the mind-body problem has been presented from the 1990s, namely in the heterodox models of situated cognition. Thus we make proposals for a better integration of verbal production environments to linguistic analysis in a cognitive perspective.

Keywords : context, cognition, social cognition, externalism, internalism, mond-body problem, reality 